\title{
Innovativer HVDC-DC-Wandler für Hochspannungs-Gleichstrom-Netze - Grundsätzliche Überlegungen zu Topologie, Regelung und elektromagnetischer Verträglichkeit
}

H. Renner OVE, U. Schichler OVE, K. Krischan, B. Deutschmann, B. Auinger, M. Horn, M. Reichhartinger, F. Vollmaier OVE

\begin{abstract}
Vermaschte Hochspannungs-Gleichstrom-Netze werden in der Zukunft bei der elektrischen Energieübertragung eine bedeutende Rolle spielen. Netzgeführte Umrichter, wie sie heute standardmäßig bei leistungsstarken HGÜ-Verbindungen zum Einsatz kommen, werden durch selbstgeführte Umrichter abgelöst und ermöglichen einen einfacheren Mehr-Stationen-Betrieb. Um einzelne, unabhängig entstandene Gleichstromsysteme mit unterschiedlicher Nennspannung verbinden zu können, ist die Entwicklung von HochspannungsGleichstromwandlern notwendig. In der vorliegenden Publikation werden grundsätzliche Überlegungen zu Topologie und Regelung angestellt und ein Demonstrator vorgestellt, der im Rahmen des institutsübergreifenden Forschungsprojektes "GriDConv" an der Technischen Universität Graz entworfen und gebaut wurde. Die Anlage ist als hybrider, kaskadierter 2-Stufen DC-DC-Wandler mit drei Zweigen ausgeführt. Damit sollen das Funktionsprinzip sowie ausgewählte Regelfunktionalitäten im Labormaßstab demonstriert werden. Die Eckdaten dieses Demonstrators wurden mit Nennspannungen von $800 \mathrm{~V}$ oberspannungsseitig, $500 \mathrm{~V}$ unterspannungsseitig und einer Übertragungsleistung von $50 \mathrm{~kW}$ festgelegt. Einen wesentlichen Punkt stellt die elektromagnetische Verträglichkeit (EMV) der Anordnung dar. Unterschiedliche Maßnahmen zur Verbesserung der EMV können mit dem Demonstrator hinsichtlich ihrer Wirksamkeit untersucht werden.
\end{abstract}

Schlüsselwörter: Hochspannungsgleichstromübertragung; HGÜ; Leistungselektronik; Gleichstromwandler; Elektromagnetische Verträglichkeit; Multil Level Converter; Leistungsregelung

\section{Innovative HVDC-DC-converter for high-voltage DC-grids-basic considerations of topology, control and electromagnetic compatibility.}

\begin{abstract}
Meshed high-voltage-DC (HVDC) grids will play a significant role in future electric energy transmission. Line commutated converters as installed by default in the recent years will be replaced by voltage source converters in the future, thus enabling a rather simple multi terminal operation. To connect HVDC installations with differing nominal voltages, the development of HVDC-DC-converters is essential. In this publication, basic considerations regarding topology and control of those devices are made and a demonstrator, designed and built within the framework of the research project "GriDConv" at Graz University of Technology, is presented. The device is designed as hybrid cascaded two-level-converter with three branches to demonstrate the principle of operation and selected control functionalities. The basic parameters are $800 \mathrm{~V}$ on the primary side, $500 \mathrm{~V}$ on the secondary side and $50 \mathrm{~kW}$ transfer capacity. Electromagnetic compatibility (EMC) plays an important role in the design. The efficiency of different methods for improving EMC can be tested with the demonstrator.
\end{abstract}

Keywords: high voltage direct current system; HVDC; power electronics; DC-converter; electromagnetic compatibility; multi level converter; active power; control

\section{Einleitung}

Die Verringerung der Importabhängigkeit Europas sowie die Einhaltung des 2-Grad-Zieles der letzten Weltklimakonferenz in Paris kann nur durch energische Maßnahmen erreicht werden. Die Lösungsansätze müssen neben der Ausgestaltung zukunftsfähiger Elektrizitätsmärkte die Energieaufbringung, Energieübertragungs- und -verteilsysteme, aber vor allem auch Effizienzmaßnahmen sowie die Mobilität betreffen. Durch die erhöhte Nutzung erneuerbarer Energien wird künftig der Anteil volatiler, dargebotsabhängiger Energie-
Renner, Herwig, Institut für Elektrische Anlagen und Netze, Technische Universität Graz Inffeldgasse 18/I, 8010, Graz, Österreich (E-Mail: herwig.renner@tugraz.at); Schichler, Uwe, Institut für Hochspannungstechnik und Systemmanagement, Technische Universität Graz, Inffeldgasse 18/I, 8010, Graz, Österreich; Krischan, Klaus, Institut für Elektrische Antriebstechnik und Maschinen, Technische Universität Graz, Inffeldgasse 18/I, 8010, Graz, Österreich; Deutschmann, Bernd, Institut für Elektronik, Technische Universität Graz, Inffeldgasse 12/l, 8010, Graz, Österreich; Auinger, Bernhard, Silicon Austria Errichtungs GmbH, Inffeldgasse 25f, 8010, Graz, Österreich; Horn, Martin, Institut für Regelungs- und Automatisierungstechnik, Technische Universität Graz, Inffeldgasse 21/B//, 8010, Graz, Österreich; Reichhartinger, Markus, Institut für Regelungs- und Automatisierungstechnik, Technische Universität Graz, Inffeldgasse 21/B/l, 8010, Graz, Österreich; Vollmaier, Franz, Silicon Austria Errichtungs GmbH, Inffeldgasse 25f, 8010, Graz, Österreich 




Abb. 1. Entwicklung der installierten HGÜ-Leistung in Europa

erzeugung jenen der bedarfsgerechten Erzeugung überwiegen. Die erforderlichen Antworten weisen einerseits auf die Entwicklung von Speichern im großtechnischen Umfang, andererseits auf eine weitere Flexibilisierung des Verbrauchs hin. Zudem stellen sich durch die weiträumige Trennung der Orte der Erzeugung und des Verbrauchs bedeutsame Transportaufgaben. Das Gesamtsystem aus Erzeugung und die Flexibilisierung des Verbrauchs werden künftig enorme Anforderungen an ein „intelligenteres" Stromsystem stellen.

Abhilfe für die steigende Zahl an Engpässen im aktuellen Hochspannungsübertragungsnetz wird durch Netzoptimierung und Netzausbau sowie durch die Errichtung von Hochspannungs-Gleichstrom-Übertragungssystemen (HGÜ) geschaffen. Netzgeführte Umrichter, wie sie heute standardmäßig bei leistungsstarken HGÜVerbindungen zum Einsatz kommen, werden in Zukunft durch selbstgeführte Umrichter (VSC, Voltage Source Converter) abgelöst. Die derzeit leistungsstärkste VSC-Anlage wird in [1] beschrieben. Diese Technologie erlaubt unter anderem einen einfacheren MehrStationen-Betrieb und die Vermaschung von HGÜ-Netzen. Die Autoren der CIGRE Broschüre 533 [2] kommen zu dem Schluss, dass aus technischer Sicht nichts gegen vermaschte HGÜ-Netze spricht. Vermehrt wird die HGÜ-Technologie neben der Punkt-zu Punkt Verbindung von galvanisch getrennten, nicht synchronen Netzen (Kontinentaleuropa - Skandinavien oder Kontinentaleuropa - England) auch eingebettet parallel zu dem bestehenden Drehstromsystem eingesetzt. Beispiele dafür sind die bereits in Betrieb befindliche Verbindung zwischen Spanien und Frankreich (Projekt INELFE) oder die geplanten HGÜ-Projekte in Deutschland (SüdLink, ULTRANET, ALEGro). Die Entwicklung der installierten HGÜ-Leistungen in Europa ist in Abb. 1 dargestellt.

Für ein vermaschtes HGÜ-Netz der Zukunft, das unter Einbeziehung einzelner HGÜ-Anlagen mit unterschiedlichen Spannungen und Technologien ein leistungsfähiges Overlay-System für das bestehende Drehstromsystem bildet, ist noch entsprechende Forschungsarbeit nötig. Dieses HGÜ-Netz der Zukunft erfordert eine Optimierung der Betriebsmittel sowie die Entwicklung neuer Technologien wie z. B. modulare Multilevel-Konverter und gasisolierte HGÜÜbertragungsleitungen.

Die Herausforderungen bei der Konzeption von vermaschten HGÜ-Netzen sind unter anderem [3]:

- Koordinierung und Festlegung der Nennspannungen,

- Schutztechnik,

- DC-Leistungsschalter

- Koordinierte Regelstrategien (lokal und übergeordnet),

- Entwicklung von HGÜ-Gleichstrom-Wandlern (HVDC-DC-Wandler),
- Entwicklung von Simulationswerkzeugen und generischen Umrichtermodellen.

Die im Rahmen der aktuell in Europa geplanten bzw. in Betrieb befindlichen Projekte (Punkt-zu-Punkt-Verbindungen) weisen unterschiedliche Nennspannungen von $\pm 320 \mathrm{kV}, \pm 400 \mathrm{kV}$ und $\pm 500 \mathrm{kV}$ auf. Eine Zusammenschaltung dieser einzelnen HGÜ-Verbindungen zu einem vermaschten HGÜ-System bedingt den Einsatz von HVDCDC-Wandlern. Die Anforderungen an derartige Wandler sind vielfältig:

- bidirektionaler Leistungsfluss,

- geringe Verluste,

- hohe Zuverlässigkeit,

- Separation von Teilnetzen im Kurzschlussfall,

- kompakte Baugröße,

- niedrige Investitionskosten.

Unter Berücksichtigung dieser Rahmenbedingungen muss festgehalten werden, dass DC-DC-Wandler-Konzepte, die für den Niederund Mittelspannungsbereich entwickelt wurden, für den Hochspannungsbereich aufgrund der extremen Spannungsbeanspruchung nicht zielführend sind. Mit den Erfahrungen von selbstgeführten Gleich- bzw. Wechselrichtern liegt der Fokus auf modularen Multilevel-Konvertern (MMC). Ein guter Überblick über eine Auswahl möglicher Topologien wird in [4] präsentiert.

\section{Topologieauswahl für einen HVDC-DC-Wandler}

In der einschlägigen Literatur existieren zahlreiche Vorschläge für die Schaltungstopologie eines HVDC-DC-Wandlers. Viele der in [4] präsentierten Schaltungsvarianten benutzen verhältnismäßig große Transformatoren zur Übertragung der gesamten oder zumindest von Teilen der zwischen den DC-Netzen auszutauschenden Leistung. Andere Topologien benötigen große, vorteilhafter Weise gekoppelte Induktivitäten, um den notwendigen Energieaustausch zwischen unterschiedlichen Zweigen der vom MMC abgeleiteten Struktur zu bewerkstelligen [5].

Im Gegensatz dazu benötigt die für die weiteren Untersuchungen ausgewählte Topologie (Abb. 2) nur relative kleine Induktivitäten, um die in den einzelnen Zweigen mit Schaltfrequenz auftretenden Komponenten der Ausgangsströme zu filtern [6]. Die Speicherung von Energie erfolgt, wie bei MMC üblich, nahezu ausschließlich innerhalb der Einzelmodule. Im Vergleich zu anderen Topologien weist die ausgewählte Variante niedrige Verluste und relativ kleine Kapazitätswerte auf. Andererseits ist unter Berücksichtigung der Spannungsfestigkeit der einzelnen Elemente die Anzahl an leistungselektronischen Komponenten höher. Diese hängt neben den absoluten Spannungsniveaus an der Ober- und Unterspannungsseite auch von dem Übersetzungsverhältnis ab, wobei der geringste Bauteilaufwand bei einem Übersetzungsverhältnis von 2 entsteht.

Im Prinzip besteht die Schaltung aus drei identisch aufgebauten Zweigen ( $a$, b und $c$ in Abb. 2). Auf der Oberspannungsseite (HV1) liegen zwei gegeneinander verriegelte leistungselektronische Schalter gegen gemeinsames Potenzial (Erde) in Serie und bilden somit Umschalter. Jeder Schalter muss die volle oberspannungsseitige Betriebsspannung $U_{H V 1}$ sperren können. Dafür werden ausreichend viele Einzelhalbleiter in Serie geschaltet, die mit den gleichen Steuersignalen versorgt werden und gemeinsam eine ausreichende Sperrfähigkeit gewährleisten.

Um bidirektionalen Leistungsfluss aber auch Separation der beiden Teilnetze im Kurzschlussfall in beide Richtungen und zudem Schwarzstart auf beiden Seiten zu ermöglichen, werden Vollbrücken für die Einzelmodule auf der Unterspannungsseite (HV2) gewählt. 


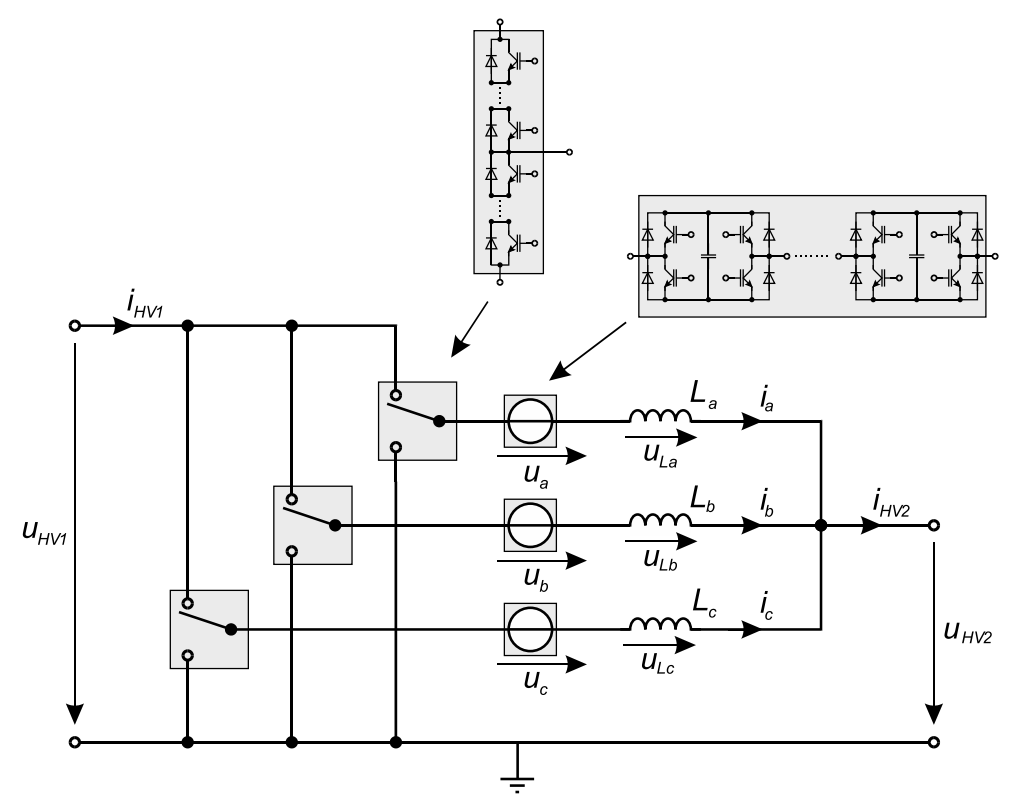

Abb. 2. Prinzip-Schaltbild der ausgewählten Topologie

Mehrere Einzelmodule werden in Serie geschaltet und bilden die einstellbare Spannungsquellen für jeden der drei Zweige. Dafür müssen auch die in Serie geschalteten Einzelmodule die Spannung $u_{H V 1}$ an der Oberspannungsseite (HV1) aufnehmen können. Die Zweigspannungen $u_{a}, u_{b}$ und $u_{c}$ können durch die Schalterstellungen in den einzelnen Vollbrückenmodulen als ganzzahliges Vielfaches der Modulspannungen eingestellt werden.

Im normalen, fehlerfreien Betrieb (kein interner Kurzschluss) muss die Summe der Ströme in den drei Zweigen den gesamten, konstanten Strom auf der Unterspannungsseite ergeben (Abb. 3). Zu jedem Zeitpunkt ist über den entsprechenden Schaltzustand der Umschalter ein Zweig mit der Oberspannungsseite und ein Zweig mit dem gemeinsamen Potenzial verbunden, während sich der verbleibende Zweig in einer Übergangsphase befindet. Diese Übergangsphase gliedert sich in den Schaltvorgang des Umschalters und die Stromaufbauphase. Während der Stromaufbauphase übernimmt der entsprechende Zweig den Strom von einem der anderen Zweige, wobei der Summenstrom konstant bleibt. Jener Zweig, dessen Strom auf null verringert wurde, kann den Schaltvorgang des Umschalters in stromlosem Zustand vornehmen, was eine einfache Serienschaltung von einzelnen Halbleiterschaltern zur Bildung der Umschalter ermöglicht. Die Zustände der drei Zweige werden zyklisch gewechselt, wobei die Schaltfrequenz im Prinzip frei wählbar ist, in der Praxis jedoch durch die zu übertragende Leistung und die Speicherfähigkeit der die Zweige bildenden Einzelmodule eingeschränkt wird. Der jeweilige Schaltzustand wird durch die Schaltfunktionen $S_{u a}, S_{u b}$ und $S_{u c}$ für den jeweils oberen Schalter (upper switch) und $S_{l a}, S_{l b}$ und $S_{\mathcal{C}}$ für den jeweils unteren Schalter (lower switch) beschrieben. Dabei steht 1 für den eingeschalteten Zustand und 0 für den ausgeschalteten Zustand.

Der Verlauf des Stromes wird durch die an den Zweiginduktivitäten $L_{a}, L_{b}$ und $L_{c}$ anliegende Spannung bestimmt. Wenn der Umschalter den Zweig a mit der Oberspannungsseite verbindet ( $S_{u a}=$ $\left.1, S_{l a}=0\right)$, ergibt sich die Spannung an der Induktivität zu

$$
u_{L a}=u_{H V 1}-u_{H V 2}-u_{a} \text {. }
$$

Wenn der Umschalter den Zweig a mit dem gemeinsamen NullPotenzial verbindet $\left(S_{u a}=0, S_{l a}=1\right)$, ergibt sich die Spannung an der Induktivität zu

$$
u_{L a}=-u_{H V 2}-u_{a} .
$$

Für die Zweige b und c gelten die Formeln in analoger Weise. Der Spannungsabfall an den Induktivitäten und damit die Stromverläufe in den Zweigen lassen sich also direkt über die Spannungen der Vollbrückenschaltungen festlegen. Die Energiebilanz der Einzelmodule bleibt dabei ausgeglichen, da - je nach Richtung des Leistungstransports - in einer Stellung des Umschalters Energie aufgenommen und in der anderen Stellung genau so viel Energie abgegeben wird, wobei nur ein Teil der zu übertragenden Energie zwischengespeichert wird.

Da die Serienschaltung der Vollbrückenschaltungen in den einzelnen Zweigen auch die maximale Spannung an der Oberspannungsseite aufnehmen kann, bleiben die Zweigströme auch bei Ausfall oder Kurzschluss von einer der beiden Seiten kontrollierbar, wodurch das Trennen der beiden Netze im Fehlerfall und der Aufbau der Spannung nach einem Ausfall ermöglicht werden.

\section{Realisierung eines HVDC-DC-Demonstrators}

Im Rahmen des institutsübergreifenden Forschungsprojektes „GriDConv" wird an der Technischen Universität Graz unter Beteiligung des Instituts für Elektrische Antriebstechnik und Maschinen, des Instituts für Elektrische Anlagen und Netze, des Instituts für Hochspannungstechnik und Systemmanagement, des Instituts für Elektronik und des Instituts für Regelungs- und Automatisierungstechnik eine Demonstrationsanlage entworfen und gebaut. Die Anlage ist als hybrider, kaskadierter 2-Stufen DC-DC-Wandler, wie er in [6] vorgeschlagen wird, mit drei Zweigen $(a, b, c)$ ausgeführt.

Damit sollen das Funktionsprinzip sowie ausgewählte Regelfunktionalitäten im Labormaßstab demonstriert werden. Die Eckdaten dieses Demonstrators wurden mit $800 \mathrm{~V}$ Nennspannung (Oberspannungsseite HV1) bzw. $500 \mathrm{~V}$ Nennspannung (Unterspannungsseite HV2) und einer Übertragungsleistung von $50 \mathrm{~kW}$ festgelegt. Dies entspricht einem Spannungsmaßstab von 1:1000 bzw. einem Leistungsmaßstab von 1:20000 für einen $1 \mathrm{GW}$-Wandler mit 800/500 kV Gleichspannung. 


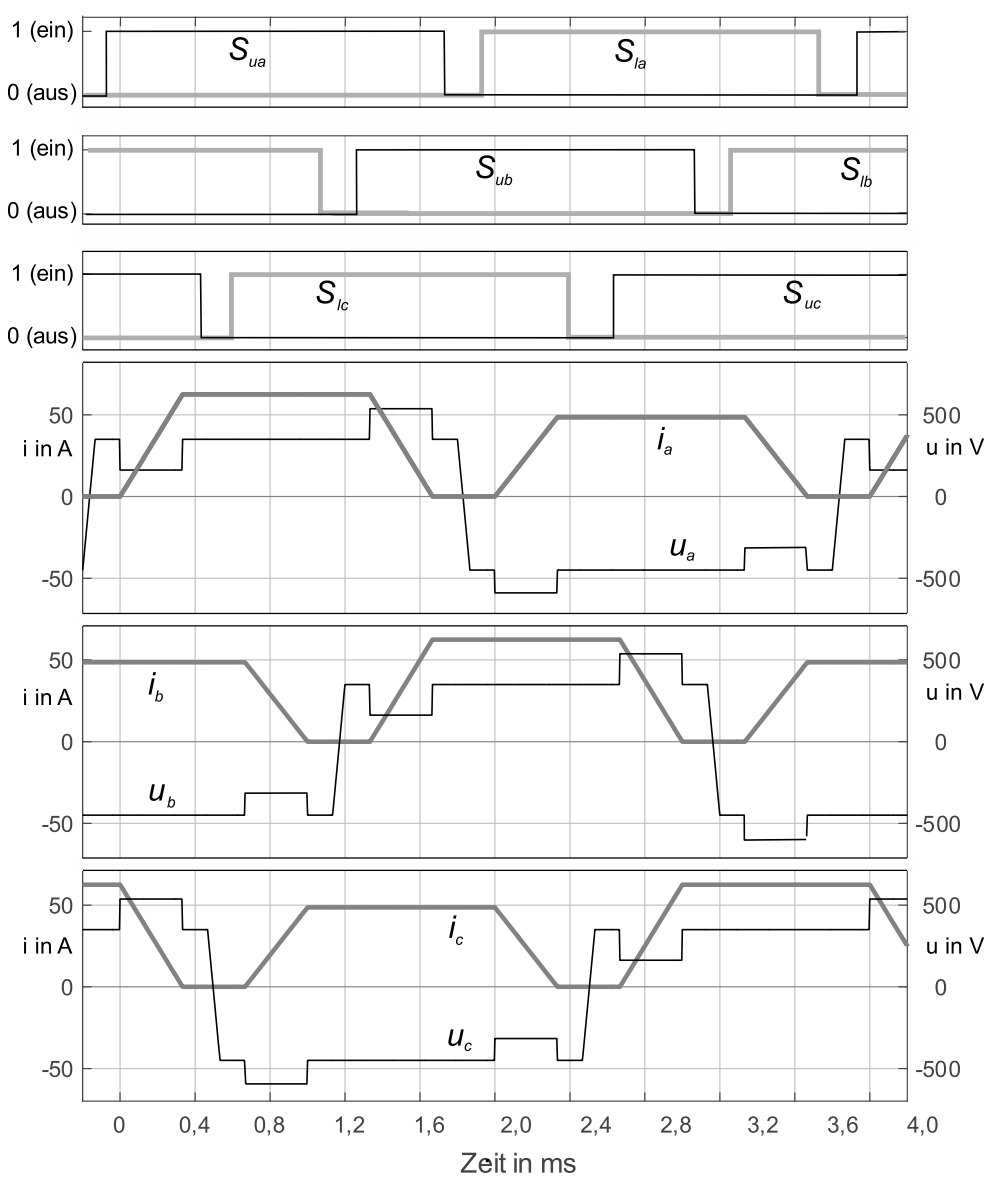

Abb. 3. Verläufe der Schaltfunktionen der oberen und unteren Serienschaltungen der IGBTs, der Sollgrößen für die Ströme in den Induktivitäten und der Spannungen der einstellbaren Spannungsquellen in den Zweigen $a, b$ und $c$

Im modular aufgebauten Demonstrator bestehen die oberen und unteren Schalter der Halbbrücken aus jeweils zwei in Serie geschalteten IGBTs und die steuerbaren Spannungsquellen aus je drei hintereinander geschalteten als Vollbrücken ausgeführten Einzelmodulen.

Ziel ist es, die Hardware, deren Ansteuerung und die implementierten Regler für die im Folgenden aufgezählten Betriebsmodi zu testen.

\subsection{Hochfahren}

- DC-Spannung liegt von beiden Seiten an: Die auszutauschende Leistung wird von der übergeordneten Leistungsregelung vorgegeben. Das Hochfahren entspricht dem Normalbetrieb bei Änderung der Führungsgröße.

- DC-Spannung liegt nur auf einer Seite an: Wieder gibt die übergeordnete Regelung den Betrieb vor, wobei diesmal der in das zu versorgende Netz einzuspeisende Strom vorgegeben wird, da eine Leistungsvorgabe bei verschwindender Spannung nicht möglich ist.

Die Hilfsspannungsversorgung für die Regelung und den Betrieb der Einzelmodule wird in weiterführenden Arbeiten untersucht werden. Nach derzeitigem Stand werden folgende Varianten untersucht werden:

- Unabhängige, externe Eigenbedarfsversorgung,

- Versorgung aus einem der Gleichspannungsnetze.
In jedem Fall wird eine Einschaltsequenz zu erarbeiten sein, die an den oben beschriebenen Situationen für das Hochfahren zu validieren sein wird.

\subsection{Normalbetrieb}

Für den Normalbetrieb sollten folgende Regelmodi möglich sein und untersucht werden:

- Leistungsregelung,

- Vorgabe Durchgangsleistung,

- Spannungsregelung,

- Leistungsregelung mit Spannungsstatik.

Diese Modi werden durch eine übergeordnete Regeleinheit realisiert, die die auszutauschende Leistung zwischen den beiden Netzen an die nächstliegende Regelungsebene weitergibt, woraus dann die Sollströme für die einzelnen Stränge abgeleitet werden können. Diese übergeordnete Regeleinheit generiert auch den Sollwert für den zu übertragenden Gleichstrom für das oben beschriebene Hochfahren.

\subsection{Fehlerfal}

\subsubsection{Externe Fehler (DC-Leitung)}

Im Fehlerfall (Kurzschluss) ist die Ausbreitung des Spannungseinbruchs zu begrenzen und die fehlerbehaftete Seite des DC-DCWandlers von der gesunden Seite zu trennen. Es erfolgt eine schnelle 
Strombegrenzung und damit einhergehend eine rasche Absenkung der Spannung auf der fehlerbehafteten Seite. Unter Annahme eines selbstlöschenden Lichtbogenfehlers wird nach Abwarten einer definierten Zeit (Entionisierung der Fehlerstelle) die Spannung rampenförmig mit einem vorgegebenen Gradienten, der die Wiederherstellung der Spannungsfestigkeit an der Fehlerstelle berücksichtigt [7], wiederhergestellt. In dieser Phase wird gegebenenfalls ein bestehender Fehler erkannt und es erfolgt eine definitive Abschaltung der fehlerbehafteten Leitung.

Dabei erfolgt die (schnelle) Strombegrenzung durch den (inneren) Stromregelkreis, während die Maßnahmen zum Löschen des Lichtbogens und des erneuten Ladens der Leitung durch die Vorgabe des auszutauschenden Gleichstroms in einer Instanz der übergeordneten Regelung realisiert werden.

\subsubsection{Interne Fehler (Konverter)}

In Abhängigkeit vorhandener Redundanzen kann der Betrieb uneingeschränkt oder mit verringerter Leistung weitergeführt werden und als defekt erkannte Komponenten können in planbaren Wartungsintervallen ausgetauscht werden. Maßnahmen zum Nutzen der vorhandenen Redundanzen zur Erkennung und Isolation von Fehlern werden in künftigen Projektphasen untersucht und weiterentwickelt werden.

Überströme in den Einzelmodulen werden auf Modulebene behandelt, indem die Treiber der Leistungshalbleitermodule Überströme erkennen und die Halbleiter ausschalten. Zusätzlich wird ein Fehlersignal generiert, das die Schalter aller Einzelmodule ausschaltet, so dass die Ströme rasch abklingen. Die Spannungen an den Kondensatoren in den Einzelmodulen werden gemessen und bei Überschreiten festgelegter Grenzen wird wieder ein Fehlersignal generiert und alle Halbleiterschalter der Einzelmodule ausgeschaltet, so dass die Spannung am Einzelmodul nicht weiter steigt.

\section{Regeltechnische Anforderungen}

Dieses Kapitel beschreibt die für einen ersten Betrieb des oben beschrieben HVDC-DC-Demonstrators notwendigen regelungstechnischen Schritte. Der Fokus des hier beschriebenen Reglerentwurfes richtet sich auf den zuverlässigen Betrieb eines Konverters, während der Betrieb mehrerer Konverter in einem gemeinsam betriebenen Netz zum aktuellen Stand des Projektes eine eher untergeordnete Rolle spielt. Die in diesem Beitrag betrachtete regelungstechnische Aufgabe besteht darin, bei gegebener DC-Spannung ober- und unterspannungsseitig eine konstante, vom Konverter zu übertragende Leistung einzustellen. Diese wird mithilfe einer im Kapitel 4.3 näher beschriebenen Stromregelung umgesetzt.

\section{1 Übergeordnete Regelung}

In der übergeordneten Regelung wird der Lastfluss im HGÜ-Netz festgelegt. Für den HVDC-DC-Wandler ergeben sich zwei mögliche Regelaufgaben:

- Spannungsregelung,

- Leistungsflussregelung

Bei der Leistungsflussregelung muss in jedem Fall eine Koordination mit den übrigen im Netz befindlichen HGÜ-Anlagen stattfinden. Im Folgenden wird auf die Leistungsflussregelung des HVDC-DCDemonstrators eingegangen.

\subsection{Bildung der Referenzströme (Zweige)}

Mit dem vorgegebenen Sollwert der Wirkleistung und der gemessenen Spannung auf der Unterspannungsseite $u_{H V 2}$ ergibt sich der Sollwert für den Strom $i_{H V 2}$.
Die in Abb. 3 dargestellten Referenzverläufe für die Zweigströme $i_{a}, i_{b}$ und $i_{c}$ in den Induktivitäten und Spannungen der einstellbaren Spannungsquellen wurden unter Einhaltung folgender Kriterien entworfen:

- Der Gesamtstrom der Unterspannungsseite HV2 entspricht der Summe der Zweigströme $i_{H V 2}=i_{a}+i_{b}+i_{c}$

- Der Gesamtstrom der Oberspannungsseite HV1 ergibt sich aus dem Skalarprodukt der Summe der Zweigströme und der Schaltfunktionen $S_{u}$ (upper switch) der oberen Serienschaltung von IGBTS

$$
i_{H V 1}=\left(\begin{array}{c}
i_{a} \\
i_{b} \\
i_{c}
\end{array}\right) \cdot\left(\begin{array}{l}
S_{u a} \\
S_{u b} \\
S_{u c}
\end{array}\right)
$$

- Die einstellbaren Spannungsquellen können keine Wirkleistung liefern oder aufnehmen

- Die Energiebilanz der Einzelmodulkapazitäten muss ausgeglichen sein

- Die Serienschaltung der IGBTs wird nur im spannungs- und stromlosen Zustand umgeschaltet

Aus den angeführten Kriterien ergeben sich theoretisch die in Abb. 3 dargestellten Zeitverläufe. Gezeigt werden die Schaltfunktionen der oberen $\left(S_{u a}\right)$ und unteren $\left(S_{/ a}\right)$ Serienschaltungen der IGBTS für eine Schaltfrequenz von $250 \mathrm{~Hz}$. Die Schaltfunktionen der einzelnen Zweige sind jeweils um ein Drittel der Periodendauer verschoben. Weiters sind die Sollgrößen für die Zweigströme $i_{a}, i_{b}$ und $i_{c}$ in den Induktivitäten sowie die einstellbaren Spannungen $u_{a}, u_{b}$ und $u_{c}$ der Vollbrücken dargestellt. Der dazugehörige Arbeitspunkt des HVDC-DC-Demonstrators wurde folgendermaßen definiert:

$$
\begin{aligned}
& u_{H V 1}=800 \mathrm{~V} \\
& u_{H V 2}=450 \mathrm{~V} \\
& i_{H V 1}=62,5 \mathrm{~A} \\
& i_{H V 2}=111,1 \mathrm{~A}
\end{aligned}
$$

\subsection{Zweigstromregelung}

Das Schema der umgesetzten Stromregelung ist in Abb. 4 dargestellt. Die Regelstrecke setzt sich aus den entsprechenden Voll- und Halbbrücken, der Ansteuerung der Schaltelemente, den Schaltelementen selbst sowie den Widerständen, Induktivitäten und Kapazitäten der einzelnen Teilstränge zusammen. Die Spannungen an der Ober- und Unterspannungsseite werden messtechnisch erfasst. Die in diesem Beitrag nicht näher betrachtete Umsetzung der von der Stromregelung berechneten Spannungen $u_{a}, u_{b}, u_{c}$ in eine entsprechende Ansteuerung der Schaltelemente wird auch als Teil der Regelstrecke interpretiert.

Die gewünschte zu übertragende Leistung wird in der Berechnung der Referenzströme $i_{a, \text { soll }}, i_{b, \text { soll }}$ und $i_{c, \text { soll }}$ berücksichtigt. Diese werden so ermittelt, dass das Produkt der Ausgangsspannung $u_{H V 2}$ mit der Summe der einzelnen Referenzströme der konstanten, vorgegebenen Leistung entspricht. Um die umgesetzte Leistung auf die einzelnen elektrischen Komponenten weitestgehend gleich zu verteilen, verändern sich die einzelnen Referenzströme mit der Zeit.

Die Stromregelung berechnet aus den Stromreferenzgrößen und den gemessenen Teilströmen $i_{a}, i_{b}$ und $i_{c}$ die Stellgrößen $u_{a}, u_{b}$ und $u_{c}$. Hierfür werden momentan für jeden Zweig separate PIRegler eingesetzt. Mögliche Verkopplungen zwischen den Teilsträngen werden derzeit noch nicht berücksichtigt. Im Folgenden ist 




Abb. 4. Regelschema für die Zweigstromregelung

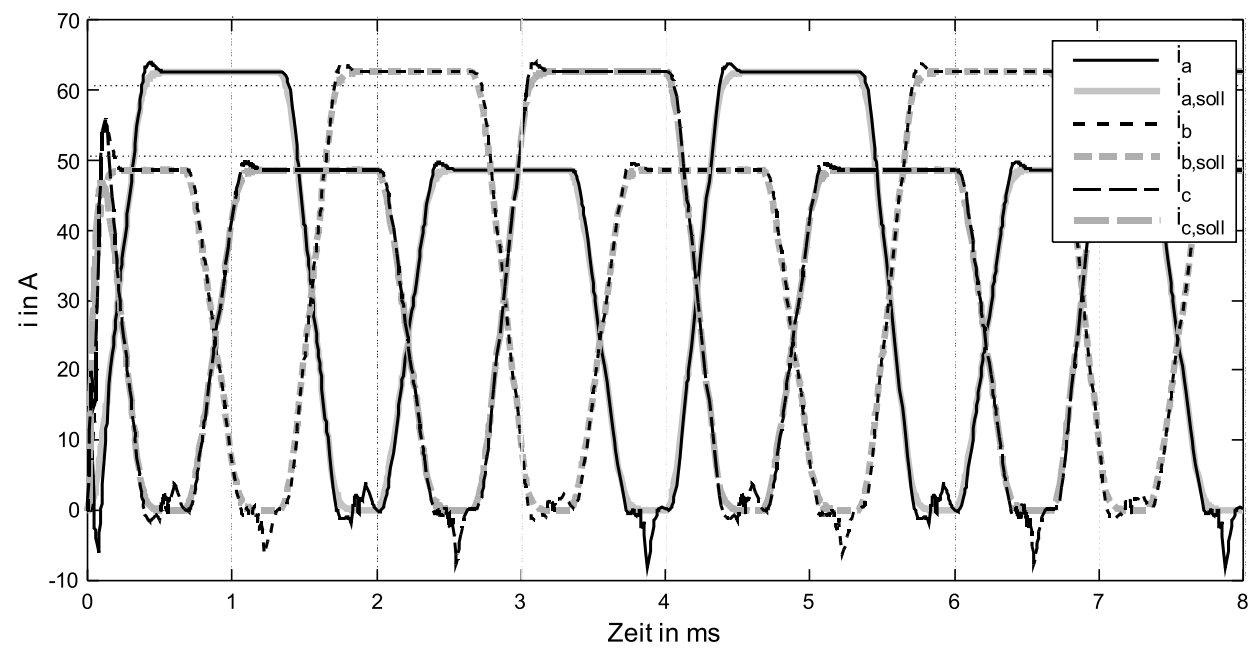

Abb. 5. Führungsverhalten der einzelnen Zweigstromregelkreise

die für die Regelung zugrunde gelegte Maschengleichung für den Zweig a dargestellt:

$$
L \cdot \frac{d i_{a}}{d t}=\left(S_{u a} \cdot u_{H V 1}-u_{H V 2}-u_{a}\right) .
$$

Die gewünschte Spannung der Vollbrückenelemente setzt sich aus Termen zur Kompensation bekannter Spannungen $\left(u_{H V 1}, u_{H V 2}\right)$ und einem gewünschten Spannungsverlauf $u_{\text {La }}$ zusammen.

$$
u_{a}=S_{U a} \cdot u_{H V 1}-u_{H V 2}-u_{L a} .
$$

Nach Kompensation verbleibt die Stromdynamik

$$
\frac{d i_{a}}{d t}=\frac{1}{L} u_{L a} .
$$

In diesem Projekt wird zur Nachführung ein PI-Regler eingesetzt. Mit dem Regelfehler

$$
e=i_{a}-i_{a, \text { soll }}
$$

ergibt sich als Ausgangssignal für den PI-Regler

$$
u_{L a}=k_{p} \cdot e+k_{i} \cdot \int_{0}^{t} e(\tau) d \tau
$$

mit den positiven Reglerparametern für den Proportionalanteil $\left(k_{p}\right)$ und den Integralanteil $\left(k_{i}\right)$. Zu dem Signal $u_{L a}$ werden anschließend die Spannungen $S_{u a} \cdot u_{H V 1}$ und $u_{H V 2}$ addiert und als Stellgröße ausgegeben.
Für erste Untersuchungen wurde ein detailliertes Simulationsmodell des Demonstrators in Matlab/Simulink implementiert. Dabei wurden die Halbbrücken, die Vollbrücken, die Stromdynamik der einzelnen Teilstränge und die Regler berücksichtigt. Die gezeigten Simulationsergebnisse wurden mit dem oben definierten Arbeitspunkt erzielt. Die zeitdiskret realisierten PI-Regelgesetze wurden alle $25 \mu$ s berechnet. Die dargestellten Simulationsergebnisse beinhalten auch das "Hochfahren" des Demonstrators.

Der zeitliche Verlauf der Sollwerte sowie die Stromverläufe für die einzelnen Zweige $\mathrm{a}, \mathrm{b}$ und $\mathrm{c}$ ist in Abb. 5 dargestellt. Abbildung 6 zeigt den Sollwert und den tatsächlichen Strom der Unterspannungsseite HV2.

Abbildung 7 zeigt die von den drei PI-Reglern berechneten Stellgrößen. Anschließend erfolgt die Umrechnung in ein Tastverhältnis welches von den Schaltelementen der einzelnen Vollbrücken in den Zweigen umgesetzt wird.

In den nächsten Entwicklungsstufen des Reglerentwurfs werden zeitlich veränderliche Eingangs- und Ausgangsspannungen berücksichtigt. Die bestehende Regelung soll also robust gegen Spannungsschwankungen am Ein- und am Ausgang sein. Zusätzlich zu der Spannungsmessung ist die Implementierung einer zuverlässigen, ausreichend schnellen Schätzung als Redundanz vorgesehen. Weiterhin soll die Vorgabe einer variablen Leistung berücksichtigt werden, da bei einer übergeordneten Leistungsregelung, die ein gesamtes Netz betrachtet, mit zeitlich veränderlichen Leistungsgrößen zu rechnen ist. 


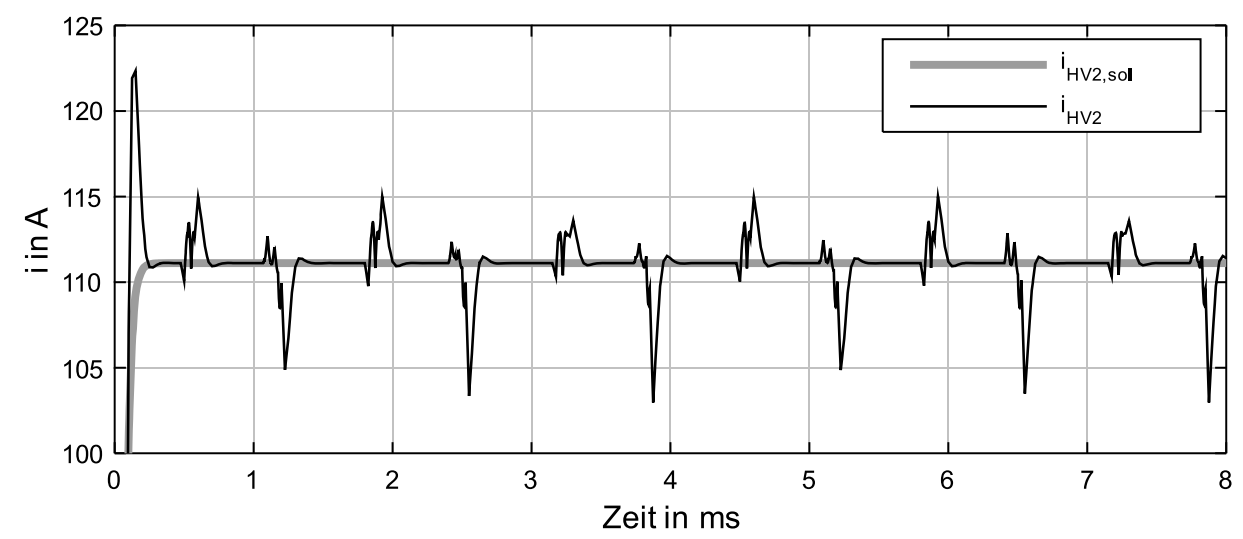

Abb. 6. Führungsverhalten des geschlossenen Stromregelkreises

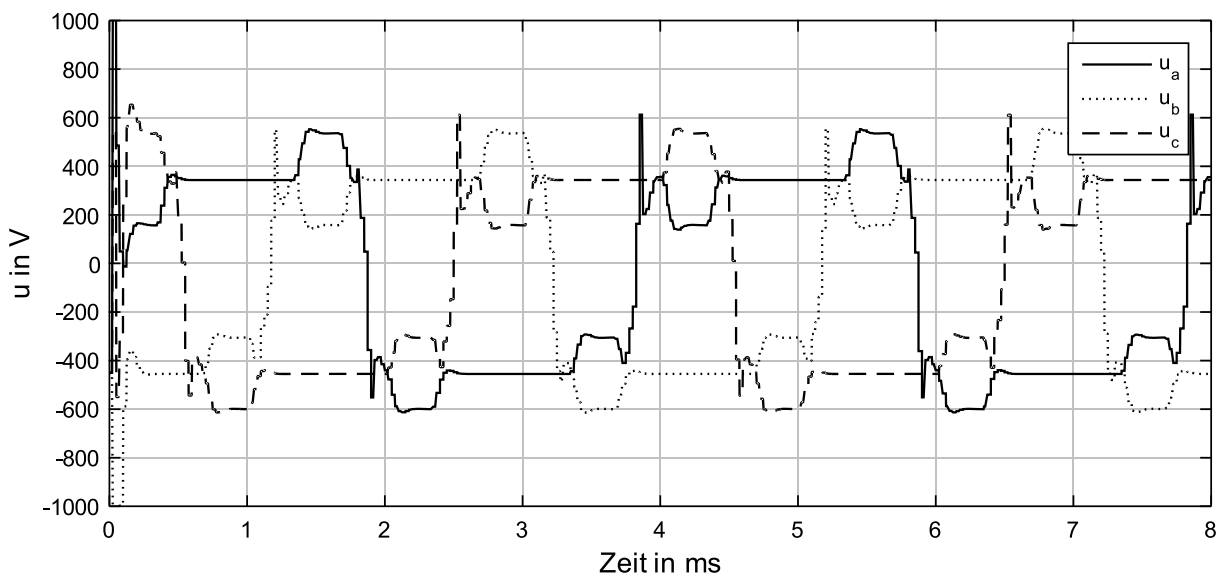

Abb. 7. Stellgrößen $u_{a}, u_{b}$ und $u_{c}$ der einzelnen Regelkreise

\section{Elektromagnetische Verträglichkeit von HVDC-DC-Wandlern}

Die elektromagnetische Verträglichkeit (EMV) der verwendeten elektrischen und elektronischen Komponenten für die HVDC-DCUmwandlung spielt eine entscheidende Rolle für den zuverlässigen Betrieb der zukünftigen Hochspannungsübertragungsnetze. Dies gilt insbesondere für die Leistungsumwandlung in HochspannungsGleichstrom-Netzen, die durch hochfrequentes Schalten von elektronischen Bauelementen und Subsystemen möglich geworden ist. Einerseits ermöglichen diese modernen elektronischen Systeme eine hohe Flexibilität für die Einstellung der Parameter der Leistungsumwandlung, können jedoch andererseits auch anfällig für elektromagnetische Störungen (EMI - Electromagnetic Interference) sein. Diese Störungen können sowohl von außen als auch von innen, d. h. von den Wandlerschaltkreisen selbst kommen, da diese durch ihre Schaltaktivität und den resultierenden Stromgradienten sehr oft als die Hauptverursacher von elektromagnetischen Störungen gesehen werden können. Ein weiterer wichtiger Aspekt ist in diesem Zusammenhang auch die Gewährleistung der störungsfreien Kommunikationsverbindung der modularen Multilevel-Konverter.

Um den fehlerfreien Betrieb von HGÜ-Netzen zu gewährleisten ist es wichtig, die EMV-Eigenschaften der verwendeten HVDC-DC Wandler genau zu analysieren. Dazu zählt nicht nur die durch das Verhalten der Schaltung erzeugten Störemissionen zu kennen und durch entsprechende Maßnahmen (Flankensteuerung, Auswahl der
Schaltfrequenzen, Gatesteuersignalregelung etc.) zu minimieren, es ist auch wesentlich, das Verhalten bei Störungen, die von außen (z. B. durch intended EMI) auftreten, zu kennen, da viele empfindliche Mess- und Regelkreise in den Konvertern verbaut sind.

Eine DC-DC-Leistungsumwandlung erfolgt meist durch hartes Schalten von hohen Spannungen mit Hochleistungsschaltern, in der Ausführung als Isolated Gate Bipolar Transistoren (IGBT) oder MOSFETs. Dieses harte Schalten mit hohen Betriebsfrequenzen erweist sich oft als Quelle von EMV-Problemen, da es einen wesentlichen Einfluss auf die erzeugte elektromagnetische Emission hat. Steile Flanken der Spannungen und Ströme in den Kommutierungskreisen erlauben es, die Effizienz der Schaltungen hoch zu halten, aber sie erzeugen meist auch hohe Störemissionen. Auf der anderen Seite schafft eine weiche Umschaltung geringe Störemission, sie bringt aber auch eine schlechte Effizienz des Wandlers mit sich. Aus diesem Grund werden geringe Störemissionen, die vom Wandler ausgehen und hohe Leistungsumwandlungs-Effizienz immer ein Kompromiss bleiben, der sorgfältig parametriert sein will. Eine Methode, um hier eine möglichst gute Reduzierung der Emission bei gleichzeitig möglichst geringen Schaltverlusten zu erzielen, bietet das Abrunden der Schaltflanken von IGBT-Leistungsmodulen durch sogenanntes „Edge-Shaping" [8]. Dabei werden insbesondere die Randbereiche der Schaltflanken (d. h. der Übergang des Signals von 0 bis $10 \%$ und von 90 bis $100 \%$ ) entsprechend geglättet um einen möglichst weichen Signalübergang zu erhalten. Wichtig dabei ist 


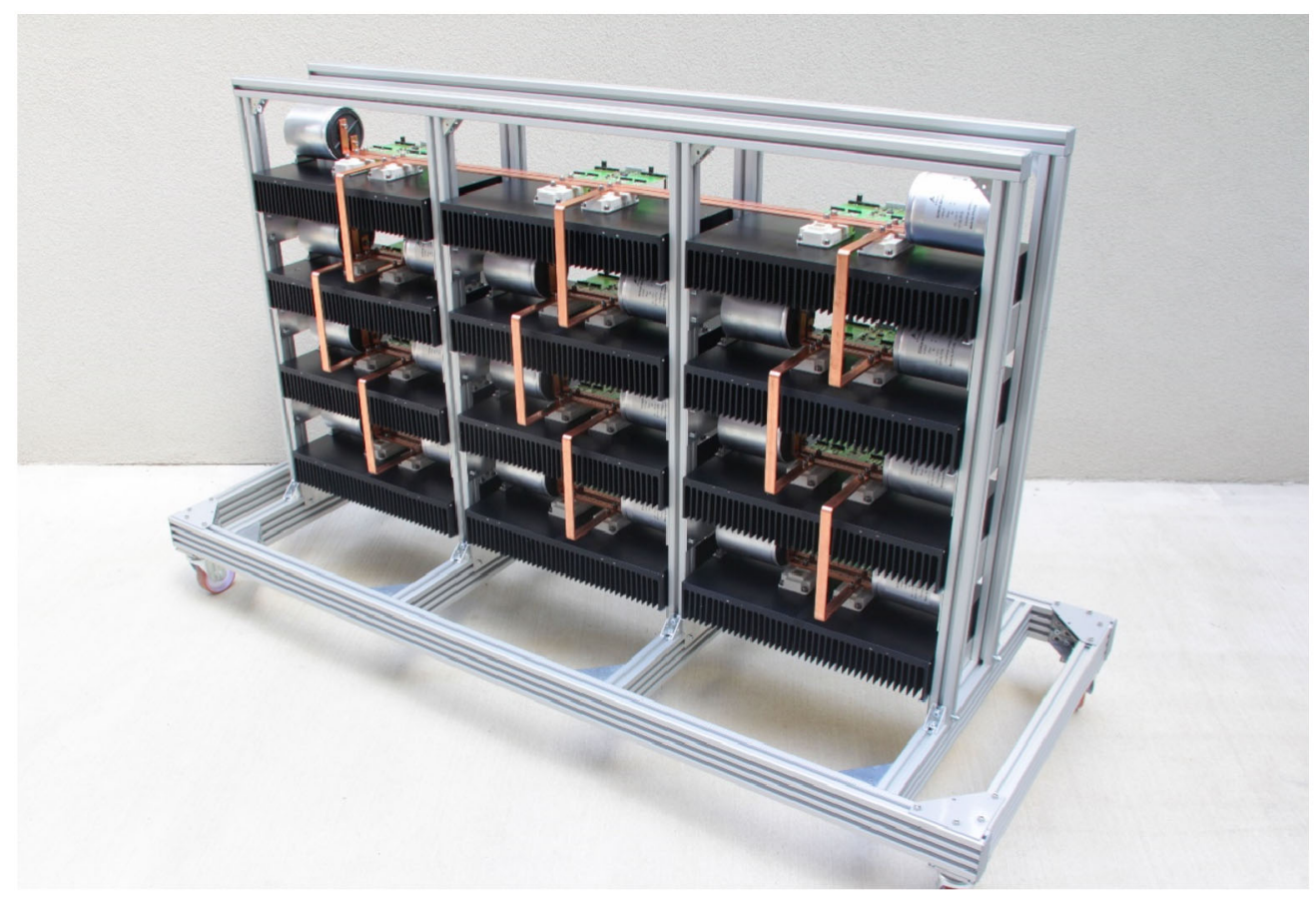

Abb. 8. Aufbau des "GriDConv"-Demonstrators; in der obersten Ebene befinden sich die durch Halbbrücken gebildeten Umschalter für die drei Zweige, in den Ebenen darunter die Vollbrückenmodule (drei je Zweig)

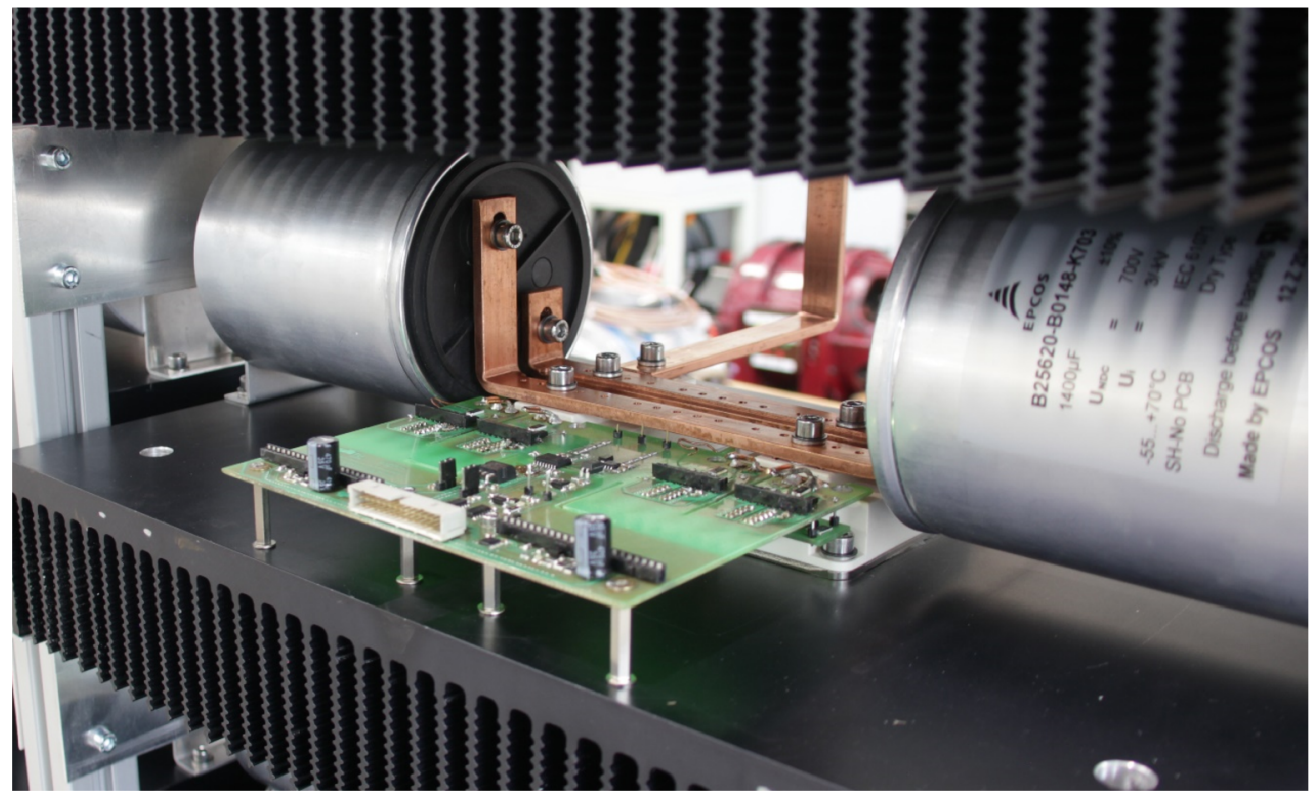

Abb. 9. Kapazitäten eines Vollbrückenmoduls und Ansteuerplatine

aber, die Gatesteuerung so auszulegen, dass die mittleren Regionen der Schaltflanken immer noch eine möglichst hohe Steilheit aufweisen damit die beim Schalten auftretenden Schaltverluste möglichst gering bleiben. Solche Techniken werden unter anderem im Forschungsprojekt untersucht. Mit Hilfe des Demonstrators sollen dabei verschiedenen Gatesteuersignalverläufe eingestellt und deren Auswirkungen auf das Schaltverhalten, insbesondere die entstehenden Störemissionen, geprüft und optimiert werden. Das Ziel ist es, eine Gateregelung zu entwickeln die unabhängig vom Lastzustand des HVDC-DC-Wandler immer ein ideales Gatesteuersignal bereitstellt, welches neben den elektromagnetischen Emissionen auch die Schaltverluste so gering wie möglich hält.

\section{Realisierter Aufbau des HVDC-DC-Demonstrators}

Abbildung 8 zeigt die realisierte Ausführung des HVDC-DC-Demonstrators. In der obersten Ebene befinden sich die durch Halbbrücken gebildeten Umschalter für die drei Zweige. In den Ebenen darun- 


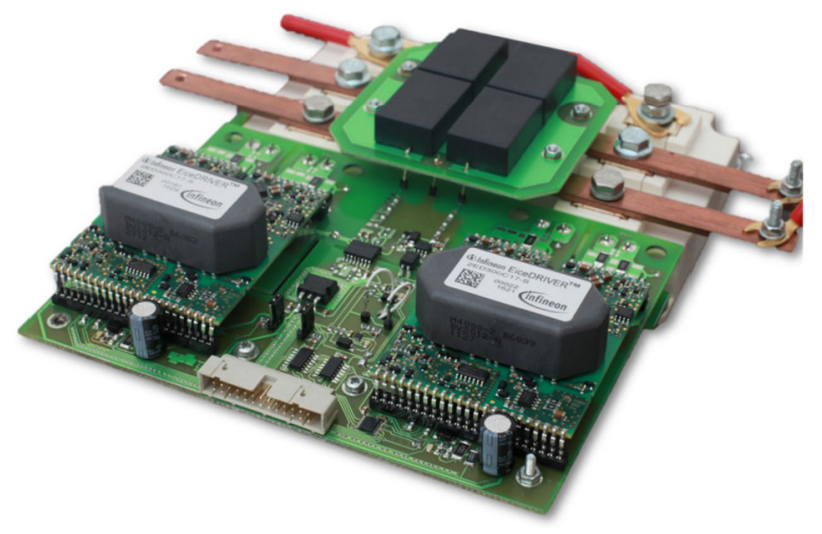

Abb. 10. Vollbrückenmodul mit Treiberschaltungen

ter sind die Vollbrückenmodule angeordnet, wobei drei Module je Zweig vorgesehen wurden.

Die Submodule beinhalten die IGBTs und ihre entsprechenden Treiber- und Schnittstellenmodule sowie die Kondensatoren zur Energiespeicherung (Abb. 9) mit zusätzlichen CeraLink ${ }^{\mathrm{TM}}$ Kondensatoren für die Hochfrequenz-Welligkeitsreduzierung sowie ein Überwachungssystem für Temperaturen und Zwischenkreisspannungen (Abb. 10). Darüber hinaus werden alle wichtigen IGBT-Steuerungs- und Schutzfunktionen wie Desaturierung, Unterspannungs-Erkennung, dynamische Überstromerkennung sowie Schutzsensoren für Überströme innerhalb des Demonstrators realisiert.

Der Betrieb des realisierten HVDC-DC-Wandlers wird durch ein FPGA-System gesteuert, das direkt mit einer Simulink-Entwicklungsplattform verbunden ist. Um die notwendigen Sicherheitsanforderungen zu gewährleisten, werden alle Steuerungs- und Überwachungssignale der Treiber- und Messplatinen durch eine zusätzliche, an einer Schnittstelle befindliche Trennbarriere, galvanisch getrennt.

\section{Zusammenfassung}

Um HGÜ-Verbindungen mit unterschiedlicher Nennspannung verbinden zu können, ist die Entwicklung von HVDC-DC-Wandlern notwendig. In der vorliegenden Publikation werden grundsätzliche
Überlegungen zur Topologie und Regelung eines transformatorlosen Wandlers angestellt. Ein im Rahmen des institutsübergreifenden Forschungsprojektes "GriDConv" an der Technischen Universität Graz entworfener Demonstrator eines HVDC-DC-Wandlers (800 V/500 V, $50 \mathrm{~kW}$ ) wird im Detail vorgestellt. Als Topologie wurde ein hybrider, kaskadierter 2-Stufen DC-DC-Wandler mit drei Zweigen ausgewählt. Mit Hilfe des Demonstrators werden das Funktionsprinzip sowie ausgewählte Regelfunktionalitäten im Labormaßstab demonstriert. Der vorgestellte innovative HVDC-DC-Wandler kann als Kernkomponente für die zukünftigen weltweiten HGÜ-Netze dienen.

\section{Danksagung}

Open access funding provided by Graz University of Technology.

Open Access This article is distributed under the terms of the Creative Commons Attribution 4.0 International License (http://creativecommons.org/licenses/by/4.0/) which permits unrestricted use, distribution, and reproduction in any medium, provided you give appropriate credit to the original author(s) and the source, provide a link to the Creative Commons license, and indicate if changes were made.

\section{Literatur}

1. Labra Francos, P., Sanz Verdugo, S., Fernández Álvarez, H., Guyomarch, S., Loncle, J. (2012): INELFE—Europe's first integrated onshore HVDC interconnection. In IEEE power and energy society general meeting.

2. CIGRE WG B4.52 (2013): Feasibility of HVDC grids. CIGRE technical brochure 533, ISBN 978-2-85873-227-2.

3. Andersen, B. R.: Overview of CIGRE activities and personal views. CIGRE SC B4 homepage.

4. Adam, G. P., et al. (2016): Review of DC-DC converters for multi-terminal HVDC transmission networks. IET Power Electron., 9(2), 281-296.

5. Norrga, S., Ängquist, L., Antonopoulos, A. (2013): The polyphase cascaded-cell DC/DC converter. In IEEE energy conversion congress and exposition, Denver, USA (S. 40824088)

6. Yang, J., He, Z., Pang, H., Tang, G. (2015): The hybrid-cascaded DC-DC converters suitable for HVDC applications. IEEE Trans. Power Electron., 30(10), 5358-5363. https://doi.org/10.1109/TPEL.2015.2420666.

7. Ebner, G., Hartmann, W., Hergt, M., Wietzel, S. (2017): Fault arc extinction and system re-start on HVDC transmission lines using LCC or VSC full-bridge converters with integrated arc recovery simulation models. In Proceedings of ACDC 2017, Manchester, UK.

8. Deutschmann, B., Illing, R., Auer, B. (2011): Edge shaping to reduce the electromagnetic emissions. In 10th Int. symposium on electromagnetic compatibility, (EMC Europe 2011), York, UK (S. 742-745). ISBN 978-1-4577-1709-3.

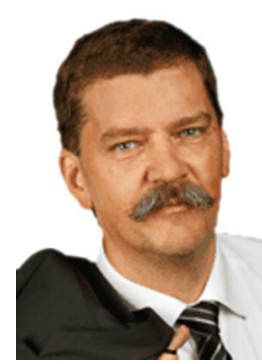

\section{Uwe Schichler}

Jahrgang 1965, ist seit 2014 Institutsleiter am Institut für Hochspannungstechnik und Systemmanagement der TU Graz. Er studierte Elektrotechnik an der Universität Hannover, Deutschland, und promovierte 1996 am Schering-Institut der Universität Hannover. Anschließend tätig für Siemens im Bereich gasisolierter Schaltanlagen und Übertragungsleitungen. Mitglied im OVE, VDE, IEEE und CIGRE. Mitarbeit in mehreren CIGRE-Arbeitsgruppen. Seit 2014 österreichischer Vertreter im CIGRE SC B3 "Schaltanlagen". Die aktuellen Arbeitsschwerpunkte liegen im Bereich Zustandsbewertung elektrischer Betriebsmittel, Teilentladungsmessungen, HGÜ-Betriebsmittel und DCIsoliersysteme. 


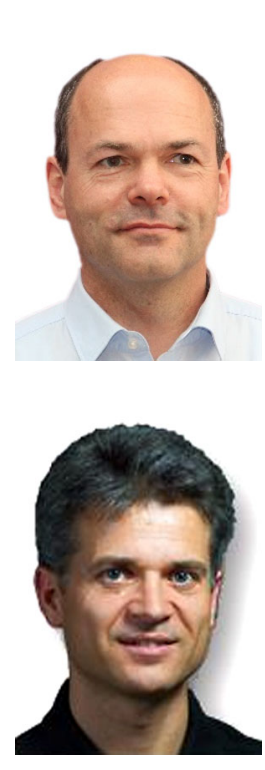

\section{Klaus Krischan}

Jahrgang 1965, Studium der Elektrotechnik an der Technischen Universität Graz, anschlieBend als Assistent am Institut für Elektrische Antriebstechnik und Maschinen der TU Graz tätig. Die Arbeitsschwerpunkte liegen im Gebiet der Leistungselektronik, insbesondere unter dem Aspekt der Verwendung in der Antriebstechnik.

\section{Bernd Deutschmann}

Jahrgang 1973, Studium der Telematik an der Technischen Universität Graz, seit 2014 Institutsleiter des Instituts für Elektronik der TU Graz, von 2000 bis 2006 bei austriamicrosystems/Österreich, von 2006 bis 2014 bei Infineon Technologies/Deutschland im Bereich der Mikroelektronik sowie auf den Gebieten der elektromagnetischen Verträglichkeit von integrierten Schaltungen und elektronischen Geräten tätig, Mitarbeit bei IEEE EMCChapter Austria.

\section{Bernhard Auinger}

Jahrgang 1977, dissertierte am Institut für Hochfrequenztechnik im Bereich Wireless Communication Test Systems an der Technischen Universität Graz im Jahr 2015. Zwischen 2005 und 2011 baute er die Gruppe für Elektromagnetische Verträglichkeitstests im Bereich Automotive bei Philips Semiconductors bzw. NXP Semiconductors auf und leitete sie. Von 2015 bis 2018 forschte er an Modellierung und Simulation im Bereich elektromagnetische Verträglichkeit für Leistungselektronik. Aktuell ist er Leiter der Research Unit „Electromagnetic Compatibility"/ im Forschungszentrum Silicon Austria Labs.

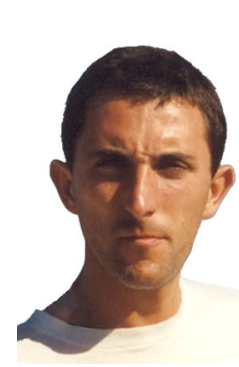

\section{Martin Horn}

Jahrgang 1966, Studium der Elektrotechnik mit dem Schwerpunkt Regelungs- und Automatisierungstechnik an der Technischen Universität Graz, 2003 Habilitation im Fach "Systemdynamik und Regelungstechnik". Von 2008 bis 2014 Professor für Mess- und Regelungssysteme an der Alpen-Adria Universität Klagenfurt, Österreich. Seit 2014 Leiter des Instituts für Regelungs- und Automatisierungstechnik an der TU Graz. Arbeitsschwerpunkte liegen in Bereich der vernetzten Regelung, der robusten Regelung und der Modellierung und Simulation dynamischer Systeme.

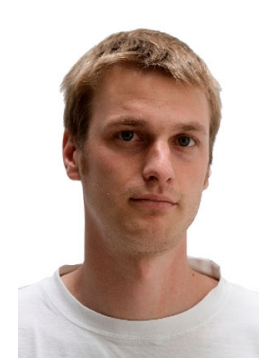

\section{Markus Reichhartinger}

Jahrgang 1980, ist Mitarbeiter am Institut für Regelungs- und Automatisierungstechnik der Technischen Universität Graz und hat 2006 das Studium der Telematik an der Technischen Universität Graz und 2011 das Doktoratsstudium der Technischen Wissenschaften an der Alpen-Adria Universität Klagenfurt abgeschlossen. Seine Forschungsinteressen beinhalten den Entwurf robuster, nichtlinearer Regelkreise.

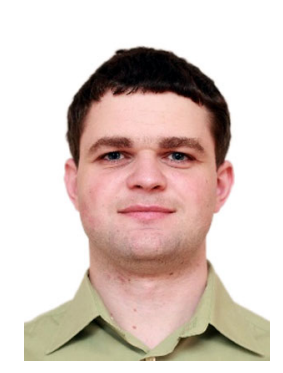

\section{Franz Vollmaier}

Jahrgang 1988, hat das Masterstudium Elektrotechnik-Wirtschaft im Jahr 2016 abgeschlossen und war während seinem Studium tätig bei Infineon Technologies/Österreich und PVP Photovoltaik. Des Weiteren ist er im Bereich der Elektronik-Entwicklung selbstständig tätig und wird im Rahmen seines Doktoratsstudiums den Fokus auf die Leistungselektronik legen. 